\title{
Permutation Machines
}

\author{
Swapnil Bhatia, ${ }^{* \dagger}$ Craig LaBoda, ${ }^{\ddagger}$ Vanessa Yanez, ${ }^{\mathbb{I}}$ Traci Haddock-Angelli, ${ }^{\S}$ and \\ Douglas Densmore ${ }^{\dagger}$
}

Department of Electrical and Computer Engineering, Boston University, Boston MA, Department of Electrical and Computer Engineering, Duke University, Durham NC, Department of Developmental, Molecular, and Chemical Biology, Tufts University, Boston MA, and International Genetically Engineered Machine, Cambridge, MA

E-mail: swapnilb@bu.edu

KEYWORDS: invertase, permutation, finite state machine, algorithm, feasible design

\section{Permuton Designer software}

We have implemented the permuton algorithms (see next section for details) into software accessible via a WWW browser at http://permuton. synbiotools.org. The software has been tested to function correctly in the Chrome and Firefox browsers (versions 50.0.2661.102 and 46.0.1, respectively). Figure 1 shows the login screen of the Permuton Designer. The login credentials may be obtained by signing up at http://welcome.synbiotools.org with an email address. Note that the application may be periodically updated, as reflected by the build date shown on the screen. Users may request new features and report incorrect behavior via email, Twitter, or

\footnotetext{
*To whom correspondence should be addressed

${ }^{\dagger}$ Department of Electrical and Computer Engineering, Boston University, Boston MA

ॠDepartment of Electrical and Computer Engineering, Duke University, Durham NC

"Tufts University

$\S_{\text {iGEM Foundation }}$
} 
a Google Group at synbiotools@gmail.com, esynbiotools, and the synbiotools group respectively. Developers may also request access to the source code or API via these channels.

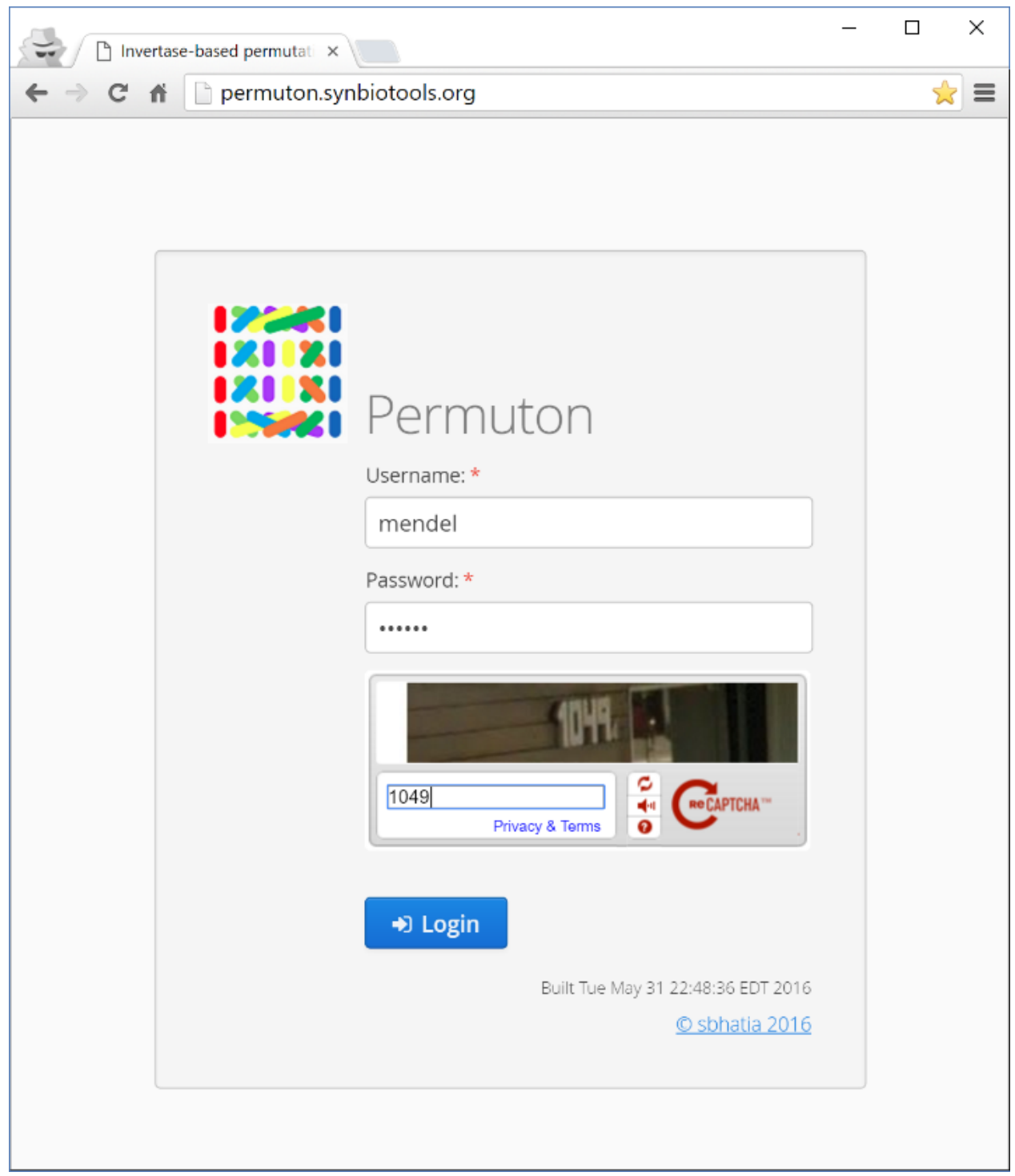

Figure 1: The Login screen for the Permuton software simulator.

Upon login, the user is presented with the screen shown in Figure 2, The user may choose from seven actions provided by the application.

- The Logout button takes the user out of the application and to the home page of the synbiotools.org website, from where the user may login to the application again. The image on the top right also takes the user to the same page. 


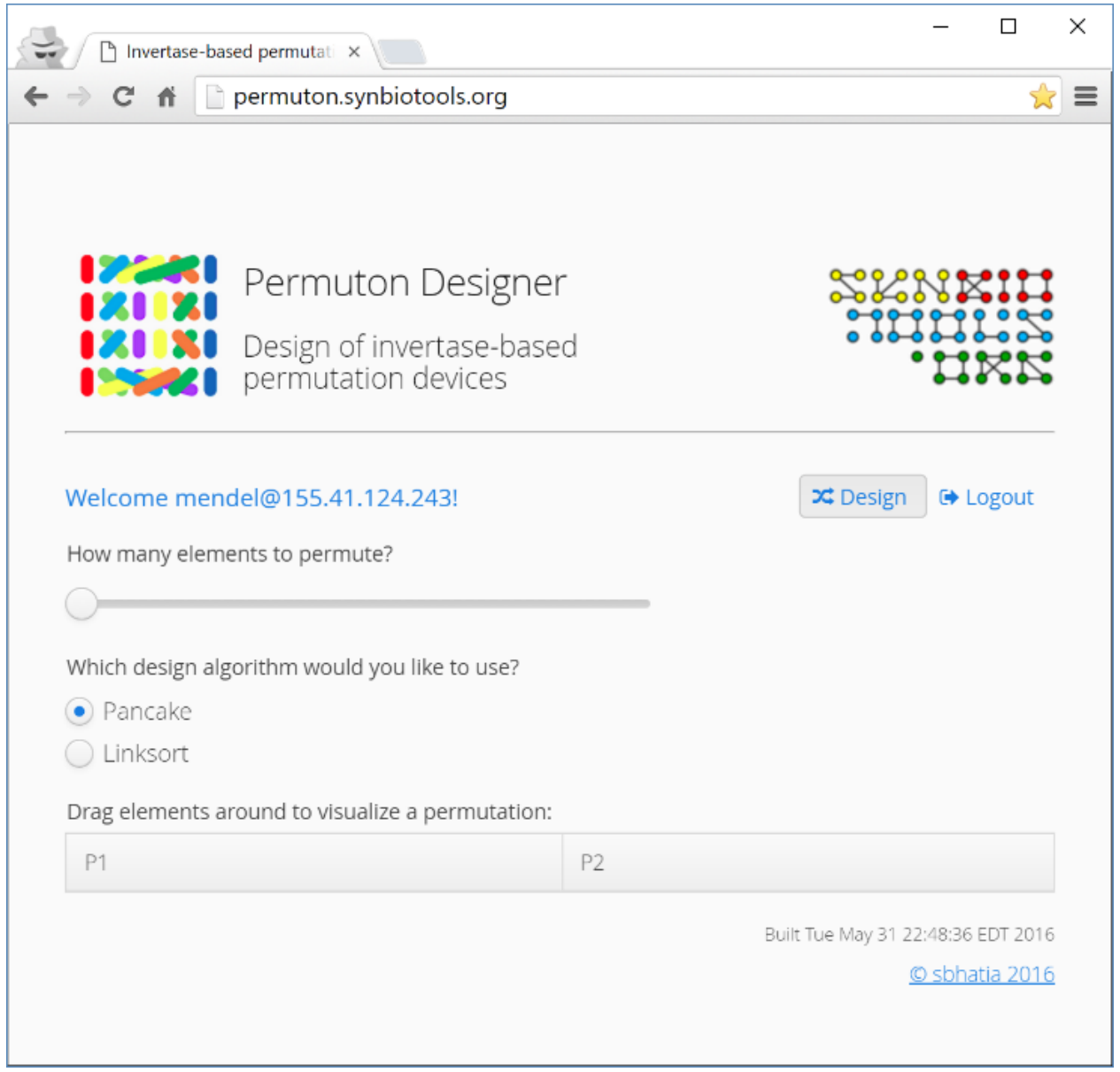

Figure 2: Upon login, the Permuton designer start screen is presented. 
- The link titled sbhatia opens a new page showing background information about the authors.

- Via the numerical slider, the user communicates the number of genetic elements to be permuted. The range is currently restricted to be between 2 and 10 to limit the transaction time for each user to a few seconds*?

- By clicking the empty circles, the user must choose one of the two permuton design algorithms: Pancake or Linksort. On doing so, the application generates a permuton using the specified algorithm and visualizes a permuton design with the specified number of elements. The design image appears in the lower portion of the application.

- The user may also specify a permutation as a target permutation to visualize. The application uses this target permutation to visualize a sequence of intermediate permutations on a path to the target permutation. These intermediates and the target permutation are visualized as images in the lower portion of the application window. The user may specify the target permutation by dragging the individual elements shown in the grey tabular row.

Figure 3 shows the state of the application when a design request is sent to it. This can be done by changing the algorithm, the number of elements in the desired permuton, or by changing the desired permutation to visualize. When the request completed, the permuton design and a path to the desired target permutation is visualized. Figure 4 shows an example of the results obtained from the Linksort algorithm on a permutation of three elements. The permuton design is shown in the first image under the permutation. Below it, a sequence of images shows the trajectory of permutations that transition the permuton from the initial permutation to the target permutation. In each image, the inversion applied is highlighted.

Figure 5 shows how the parameters presented to the application in Figure 4 can be changed to obtain a new design. As shown in Figure 5, the algorithm is changed to Pancake, which would

\footnotetext{
*We note that this is not a shortcoming of the algorithms presented above, but of the image generation technology used to generate the final output image.
} 


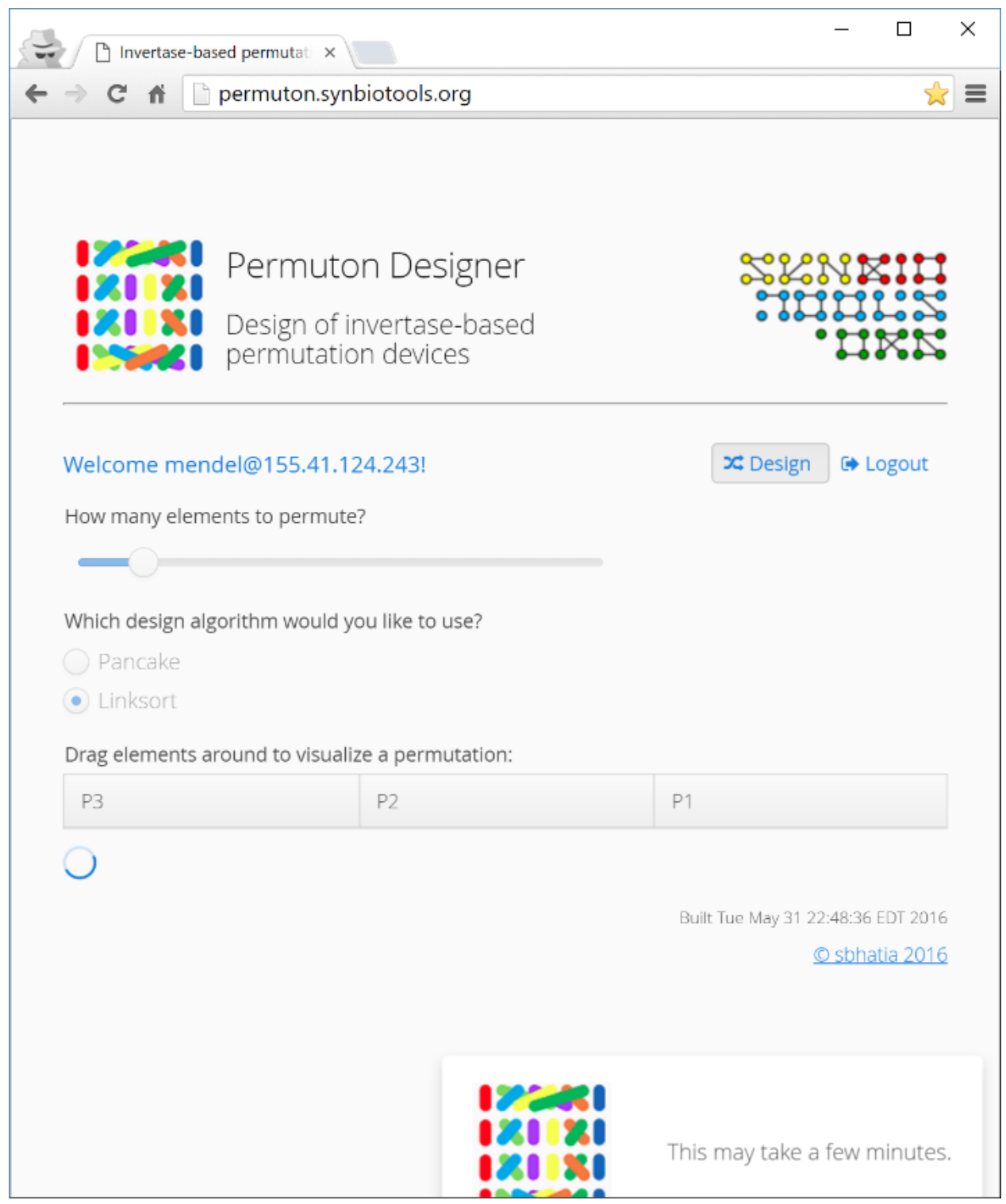

Figure 3: On choosing a new number of elements to permute, or a permuton algorithm, the designer begins to generate a new design. The user is informed that this may take several seconds to minutes. We note that a majority of the time is spent in generate the image visualization file, rather than in executing the algorithm.

have resulted in the same Pancake permuton shown in Figure 5. Then, the target permutation to visualized was changed from "3 21 " to "2 13 " which resulted in the new trajectory shown. 


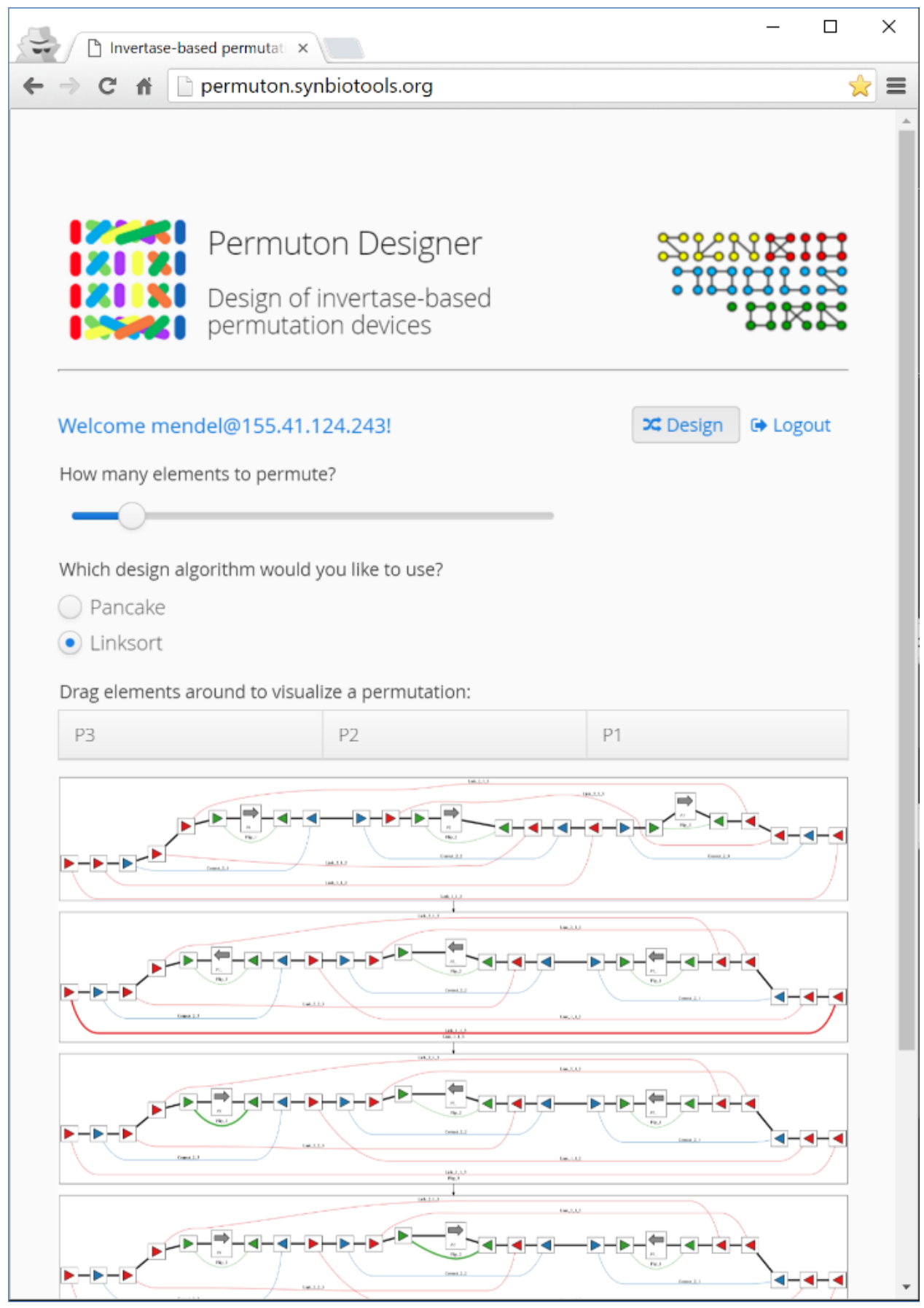

Figure 4: A Linksort permuton of three elements is generated by the application. A trajectory to the target permutation " 321 " is also visualized by the application. 


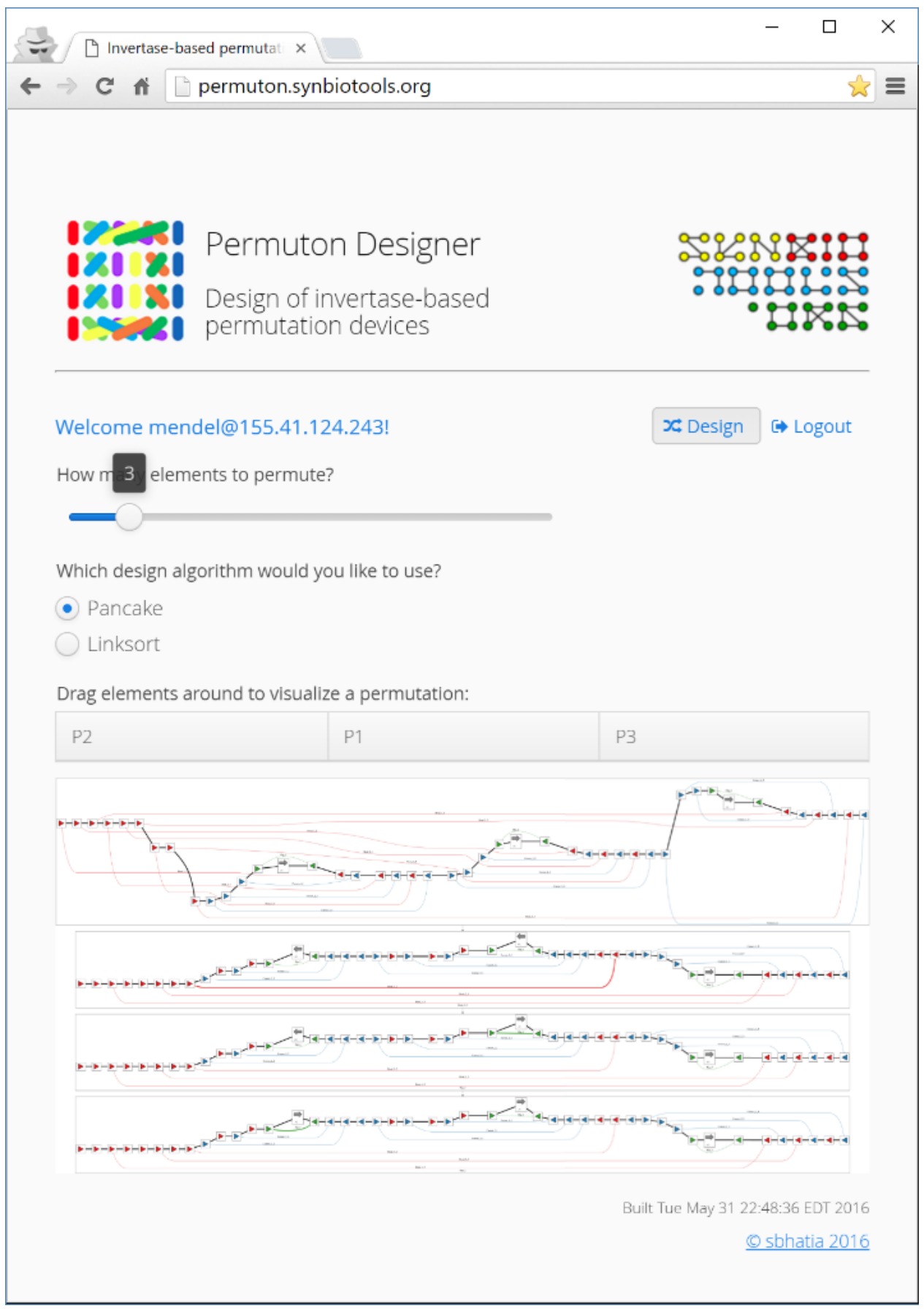

Figure 5: A pancake permuton of three elements is generated by the application. A trajectory to the target permutation " 213 " is also visualized by the application. 


\section{Algorithms}

The following document outlines the design and key generating algorithms for each of the permuton architectures. Within these algorithms, the DNA sequences representing the $\mathrm{n}$ different elements of a permuton are denoted as $e_{x}$, where $x=1$ to $n$. DNA sequences representing invertase sites are denoted by their type, e.g. head, link, correct, and flip, along with a set of indexing subscripts. A pair of invertase sites have the same type name and subscripts, with the right site having an additional apostrophe following the name, e.g. $\operatorname{Head}_{1,1}$ and $\operatorname{Head}_{1,1}^{\prime}$.

The design algorithms for Pancake and Linksort permutons assemble the front of the permuton separately from the rest of the permuton. This front portion of the construct is denoted as $\tau$. The key generating algorithms keep track of both the target permutation and the temporary current permutation (while determining the key). These two permutations are represented by $\gamma$ and $\sigma$ respectively. The key itself is represented as $\phi$, which stores the series of invertases that must be applied to the original construct to achieve $\gamma$.

\section{Pancake Algorithms}

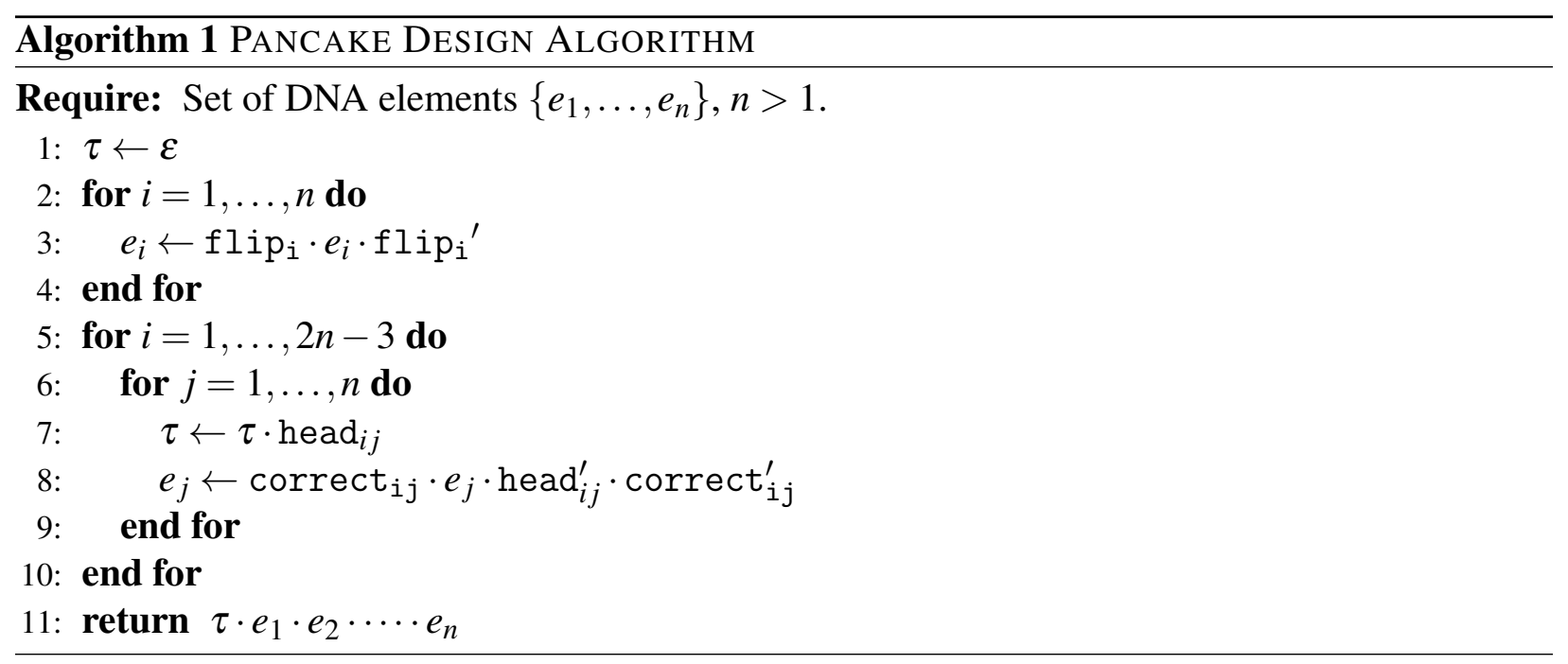




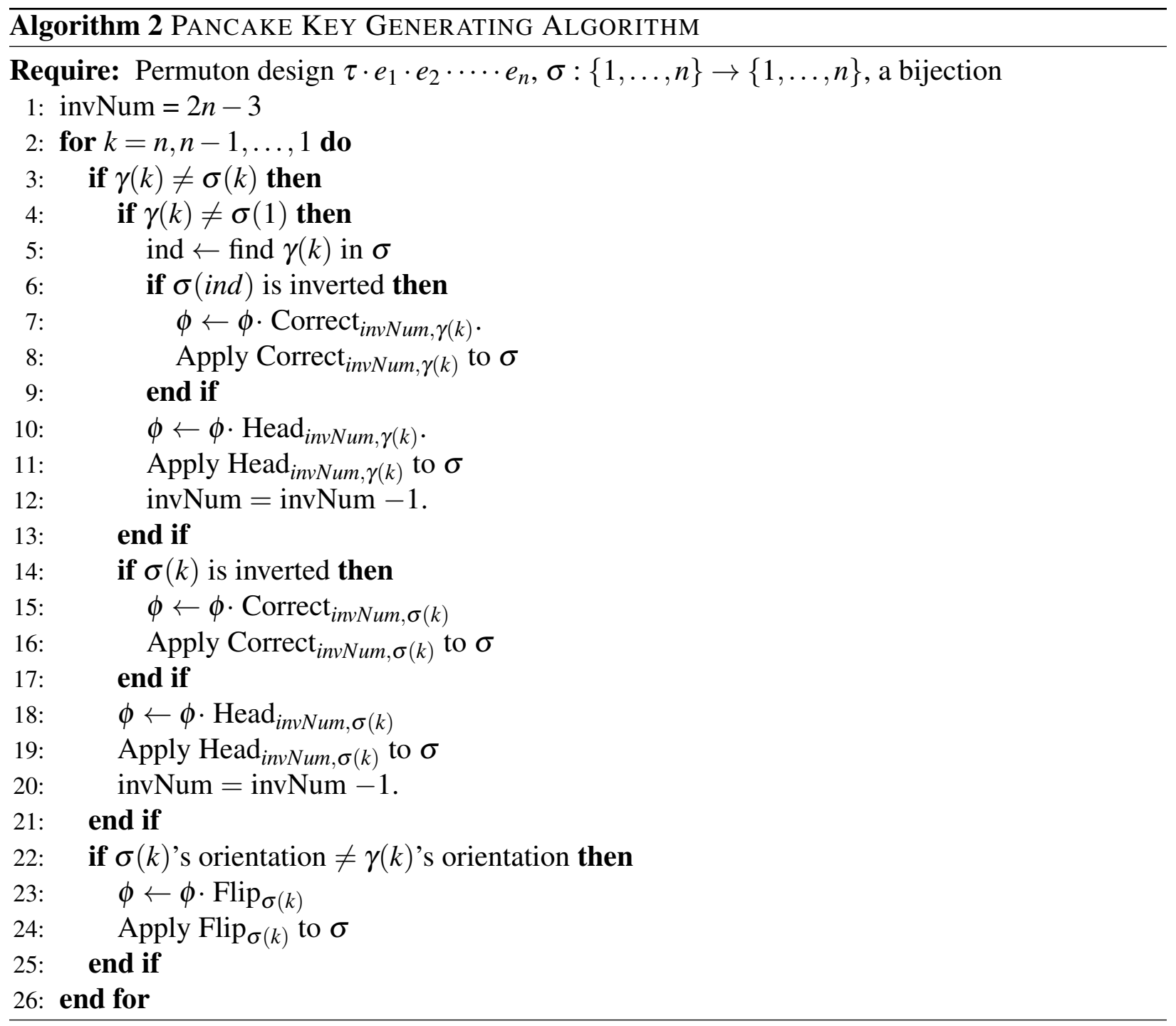




\section{Linksort Algorithms}

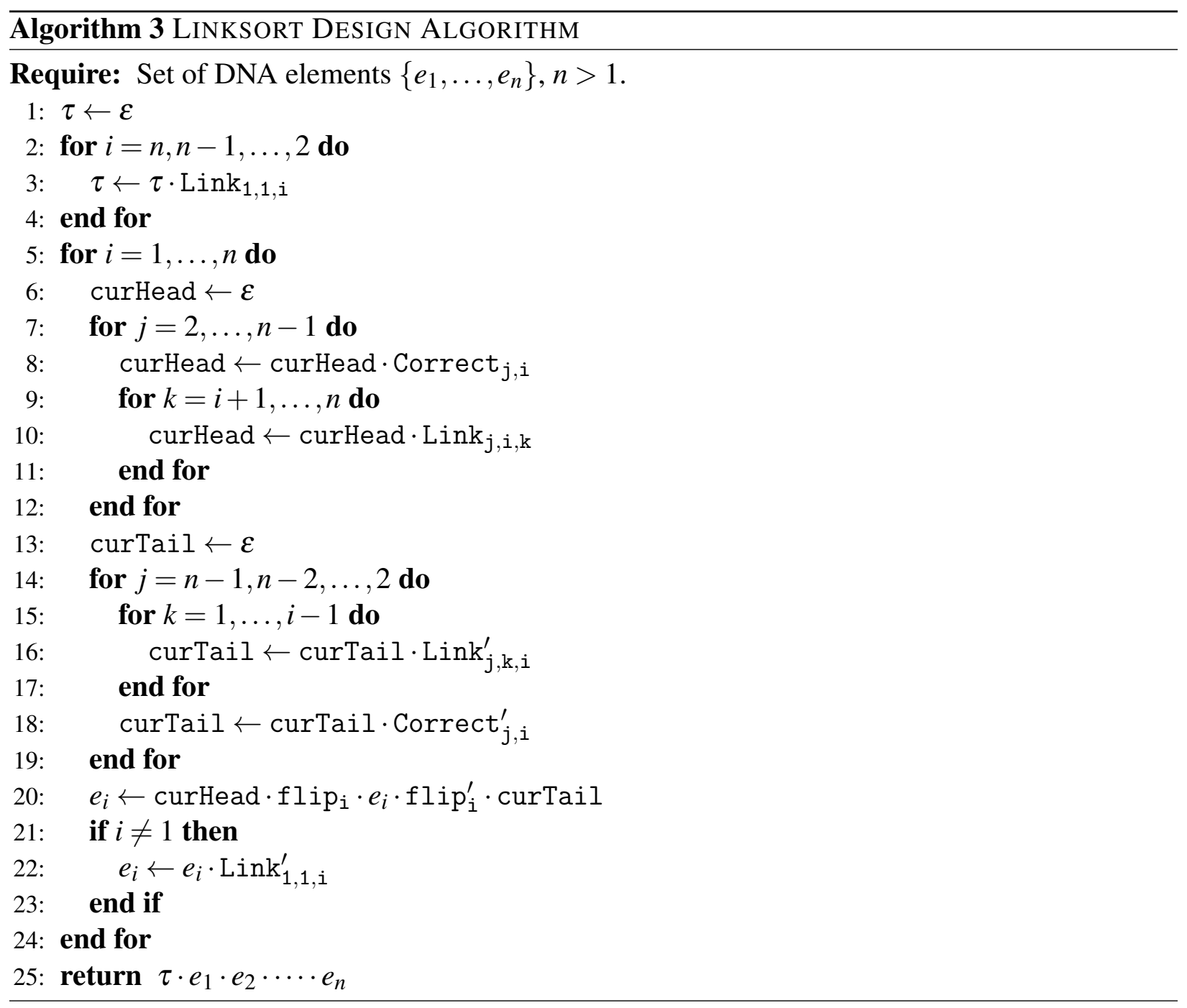




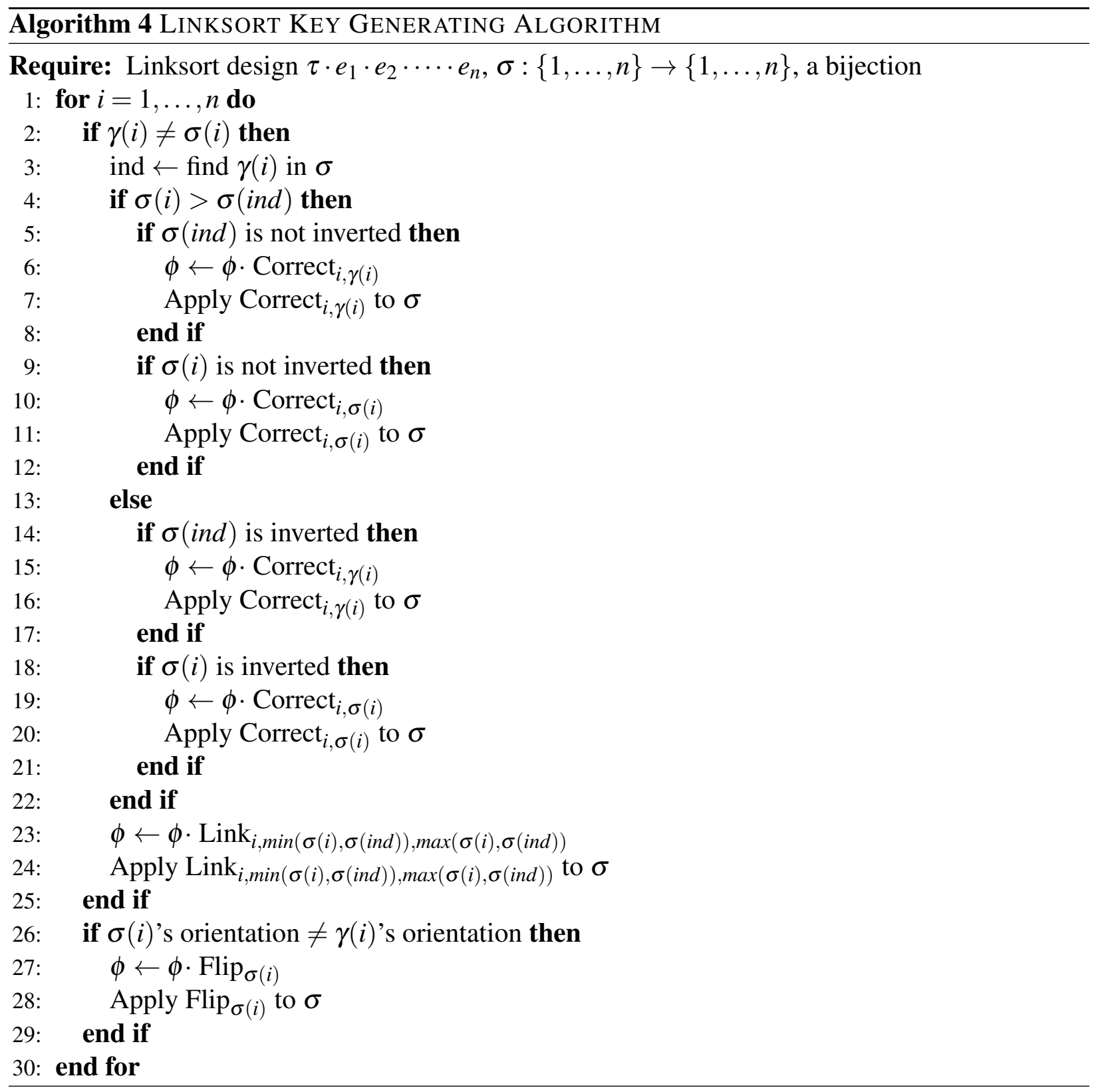

